\title{
La Unión Europea como paradigma civil y político para el siglo XXI
}

\author{
The European Union as a Civil and \\ Political Paradigm for the 21st Century
}

\author{
Marta Postigo Asenjo
}

Universidad de Málaga martapostigo@uma.es

DOI: http://doi.org/10.15366/bp2019.20.009

Bajo Palabra. II Época. No20. Pgs: 161-180 
Los temas abordados en el presente texto forman parte del trabajo realizado como miembro del Proyecto del Plan Nacional de Investigación I+D+i Civic Constellations II (CivCons): Debating Democracy and Rights (FFI2014-52703-P, 2015-2018). Estoy muy agradecida a las Profesoras María G. Navarro y Griselda Gutiérrez por la oportunidad de presentar una versión previa en el Simposio por ellas coordinado sobre "La desinstitucionalización de la política”, en el 56 Congreso Internacional de Americanistas (ICA) 2018, que se celebró en la Universidad de Salamanca el 19 y el 20 de julio de 2018, así como a la Profesora Concha Roldán Panadero por ponerme en contacto con ellas.

\section{नेरrणy}

\section{Resumen}

La UE constituye una novedad institucional y cívico política que plantea interrogantes a la teoría política. Por ello, resulta relevante tenerla en cuenta para pensar la ciudadanía y la comunidad política del siglo XXI, en un mundo globalizado y cosmopolita. No obstante, el proyecto comunitario, y su logro más significativo desde el punto de vista cívico, que es la ciudadanía europea, afrontan significativos desafíos ante el auge de los populismos y de los nacionalismos, como pone de relieve el Brexit. El presente artículo tiene por objetivo destacar la singularidad política de la UE y señalar los desafíos que plantea ésta a la teoría política, así como los logros e incertidumbres de la ciudadanía comunitaria.

Palabras Clave: UE, doble ciudadanía, teoría política, nacionalismo, cosmopolitismo.

\section{Abstract}

The EU represents an institutional and ontological novelty that raises conceptual challenges to political theory. Indeed, the $\mathrm{EU}$ is key to understanding the civil and political community of the 21 st century, suitable for the globalized world. Yet, the European project, and its main civic achievement, that is, Union citizenship, faces deep challenges in light of nationalist and populist discourses expanding all across Europe, as the case of the so-called Brexit shows. This articles aims at highlighting the political singularity of the EU and underlining the challenges it poses to political theory, stressing the reach and shortcomings of Union citizenship.

Keywords: EU, double citizenship, political theory, nationalism, cosmopolitanism. 


\section{Introducción: la relevancia de la UE para pensar el futuro de la comunidad cívico-política}

La Unión Europea (UE) constituye una novedad institucional y cívico-política que carece de precedentes y equivalentes históricos. Ésta no es un estado nacional, pero tampoco se trata simplemente de una organización internacional. Entre otros aspectos singulares, la UE es la única organización política supranacional que tiene un Parlamento propio democráticamente electo por votación directa de la ciudadanía. Definir su estructura jurídico-constitucional multinivel, su régimen de gobierno y su doble dimensión interestatal y supranacional supone un reto para la teoría política.

Es precisamente dicho carácter elusivo y controvertido lo que convierte a la UE en una entidad estimulante para la reflexión académica y en candidata a ser tomada como referencia para pensar el paradigma político del siglo XxI.

Desde la segunda mitad de siglo XX, la soberanía de los estados ha dejado de ser absoluta y coexiste con las instituciones supranacionales de toma de decisiones y con una sociedad civil organizada en redes y en asociaciones transnacionales.

Vivimos en un mundo globalizado crecientemente intercomunicado e interdependiente, en el que el aislamiento y la independencia plena de los estados parece cosa del pasado. Por ello, a pesar de sus limitaciones y deficiencias, la UE puede ofrecer una respuesta a la crisis política y ontológica del estado-nacional.

Ciertamente, afianzar los valores liberal-democráticos y el modelo cosmopolita de ciudadanía europea requiere compromisos a medio y largo plazo. No cabe duda, de que el proyecto comunitario se enfrenta a profundos desafíos ante el auge de los nacionalismos y de los movimientos antieuropeos como el Brexit.

Sin embargo, tales tendencias expresan el antagonismo que caracteriza a nuestro siglo: junto a la porosidad y a la apertura de las fronteras que definen al proceso de globalización, se reproducen los discursos nacionalistas y las actitudes chovinistas, se elevan murallas, no sólo físicas, sino también simbólicas, para dividir a comunidades e impedir los flujos migratorios, y prolifera el fanatismo religioso.

En las próximas páginas ofrezco una reflexión sobre los desafíos conceptuales que la UE plantea a la teoría política. El objetivo es reflejar su novedad y su singularidad ontológica e institucional, y señalar cómo se redefine el lenguaje democrático en el marco de la estructura multinivel y plurinacional europea. 
Junto a ello, pongo de relieve los logros y los desafíos políticos y legales de la ciudadanía de la Unión: un proyecto cosmopolita que puede servir de modelo para pensar el paradigma político del siglo xxI, pero que, sin embargo, se enfrenta a profundos retos ante las retóricas nacionalistas y antieuropeas, así como a causa de una integración minimalista en lo que respecta a la cohesión social.

Como conclusión planteo la reflexión más amplia sobre la tensión entre la dimensión universal de la ciudadanía y la soberanía democrática.

\section{2. ¿Qué es la UE? La cuestión ontológica y el impacto de la UE en la teoría política}

A COMIEnzos DEL SIGLO XXI vivimos en un planeta globalizado e intercomunicado gracias a las tecnologías de la comunicación; en un mundo, en consecuencia, más homogéneo e interconectado ${ }^{1}$. Los estados nacionales, que han constituido el marco político e institucional de la ciudadanía y de la democracia modernas, han cedido parte de su soberanía a organizaciones políticas supranacionales y a instituciones internacionales, y las fronteras de la ciudadanía se han transformado y ampliado. Puede hablarse de la emergencia de una sociedad civil global y transfronteriza ${ }^{2}$.

En dicho contexto, la UE representa un espacio y un experimento político que puede resultar paradigmático para pensar el futuro de la ciudadanía y de la soberanía democrática en el mundo globalizado y en un escenario cosmopolita.

Ciertamente, la integración europea plantea un desafío conceptual a la teoría política. Por ello, junto a la reflexión sobre las ventajas e inconvenientes que tiene la UE para la soberanía democrática o para la ciudadanía conviene prestar atención al desafío que ésta plantea al lenguaje político.

\footnotetext{
${ }^{1}$ Ulrich Beck, Was ist Globalisierung? Irrtümer des Globalismus - Antworten auf Globalisierung. Frankfurt am Main: Suhrkamp, 1997; David Held y Anthony McGrew Globalization/Anti-Globalization. Cambridge: Polity Press. 2002; Thomas L. Friedman, The World is Flat. A Brief History of the Twenty-First Century. Nueva York: Picador/ Farrar, Straus and Giroux, 2005.

2 Helmut K., Anheier, Mary Kaldor y Marlies Gasious, eds., Global Civil Society 2005/6. Londres: SAGE Publications, 2006; Seyla Benhabib, The Rights of Others. Aliens, Residents and Citizens. Cambridge: Cambridge University Press, 2004; Seyla Benhabib, Ian Shapiro y Danilo Patronovic, eds., Identities, Affliations, and Allegiancies. Cambridge: Cambridge University Press, 2007; Seyla Benhabib, Another Cosmopolitanism. Robert Post, ed. Oxford University Press, 2006; Manuel Castells, The Rise of the Network Society. The Information Age: Economy, Society and Culture. Vol. I. Cambridge, MA; Oxford, UK: Blackwell, 1997; Mary Kaldor, Global Civil Society An Answer to War. Cambridge: Polity Press, 2003; John Keane, ¿Global Civil Society? Cambridge: Cambridge University Press, 2003.
} 
Tras más de seis décadas de integración no existe consenso sobre qué tipo de organización política constituye la UE. Ésta se define en ocasiones como una organización federal, cuasi-federal o confederal, pero dicha categorización no logra un consenso académico ni político suficiente ${ }^{3}$.

En otras fuentes y discursos, se recurre a una terminología política alternativa y se define a la Unión como un sistema funcionalista de integración y de gobernanza interestatal ${ }^{4}$, como una estructura jurídica multinivel ${ }^{5}$, una constelación postnacional $^{6}$ o cosmopolita ${ }^{7}$.

Además del desacuerdo que suscitan tales categorías, frecuentemente se confunden y se mezclan los juicios descriptivos y normativos cuando se trata de definir la ontología política comunitaria, de forma que resulta casi imposible separar la cuestión sobre el ser de la Unión -qué es la UE empíricamente- de la del deber ser -en qué tipo de politeia debe convertirse la UE-.

Ello puede observarse cuando se aborda el régimen político y de gobierno comunitario. A pesar de que el Tratado de la Unión Europea (TUE) establece que la Unión se funda en el principio de la democracia representativa (Título II, artículo 10), resulta cuanto menos discutible qué tipo de democracia, si puede llamarse así, tiene lugar a nivel comunitario ${ }^{8}$. En la Unión existen al menos tres canales de representación: la representación directa de la ciudadanía europea que tiene lugar en el

\footnotetext{
${ }^{3}$ Por ejemplo, diferentes perspectivas y posiciones con respecto al federalismo europeo pueden encontrarse en Michael Burgesss, Federalism and the European Union: The Building of Europe, 1950-2000. Londres: Routledge, 2000; R. Castaldi, "A Federalist Framework Theory on European Integration", Centro Studi Federalismo, 2007, 1-93; S. Dosenrode, "Federalism Theory and Neo-functionalism: Elements for an Analytical Framework", Perspectives on Federalism, Vol. 2, Issue 3 (2010), 1-28; Daniel J. Elazar, Exploring Federalism. Londres: The University of Alabama Press, 1987; Robert Schuman, Declaración de Robert Schuman, 9 de mayo de 1950, accesible en línea en la página web oficial de la UE https://europa.eu/european-union/about-eu/symbols/europe-day/ schuman-declaration_es (05/05/2018).

${ }^{4}$ Philippe Schmitter, "What Is There to Legitimize in the European Union...and How Might This be Accomplished?” European University Institute (enero, 2001), en-línea: http://www.eui.eu/Documents/DepartmentsCentres/SPS/Profiles/Schmitter/LegitimizeEU.pdf, (08/11/2012); Philippe Schmitter y Alexander H. Treschel, coords., "The Future of Democracy in Europe: Trends, Analysis and Reforms". A Green Paper for the Council of Europe. Council of Europe, 2007, en línea. http://www.thefutureofrepresentativedemocracy.org/files/pdf/resources/schmitter_thefutureofDemocracyinEurope.pdf (09/11/2012).

5 Mario Fabbrini, "Transatlantic Constitutionalism: Comparing the United States and the European Union", European Journal of Political Research, 43 (2004), 547-569; Mario Fabbrini, "The European Multilevel System for the Protection of Fundamental Rights: A 'Neo-Federalist Perspective', Jean Monnet Working Paper, 15/10 (2010).

${ }^{6}$ Jürgen Habermas, Die postnationale Konstellation: Politische Essays. Frankfurt: Suhrkamp Verlag, 1998.

7 Ulrich Beck, Cosmopolitan Vision. Cambridge: Polity Press, 2006; Ulrich Beck y Edgar Grande, Cosmopolitan Europe. Cambridge: Polity Press, 2007.

8 Oliver Costa, "The Coming of Age of the European Parliament", Journal of European Integration, 8 de marzo de 2017, http://www.tandfonline.com/doi/full/10.1080/07036337.2017.1297021 (08/03/2017); Diego Praino, "A New System of Government? Defining the Confidence Relationship of the EU Model", Journal of European Integration, 01 de Febrero de 2017, http://www.tandfonline.com/doi/full/10.1080/07036337.2017.1281264 (08/03/2017).
} 
Parlamento Europeo (artículo 10.2 TUE); la indirecta, que llevan a cabo los Jefes de Estado y de Gobierno en el Consejo Europeo y los ministros en el Consejo, quienes son democráticamente responsables, "bien ante sus Parlamentos nacionales, bien ante sus ciudadanos" (artículo 10.2 TUE); y en los procesos representativos y electorales que tienen lugar en los Estados Miembros?.

En consecuencia, resulta pertinente preguntarse por la existencia de un demos comunitario putativo que estaría representado en el Parlamento Europeo ${ }^{10}$, o si es más adecuado definir a la UE como una demoi-cracia, esto es, como un conglomerado de pueblos (demoi) y de Parlamentos soberanos ${ }^{11}$. A este respecto, la diferencia entre los criterios normativos y puramente descriptivos se desvanece cuando se discute sobre la demoicracia europea.

Por otra parte, en los últimos años, y particularmente a raíz de la crisis económica, se han acentuado las críticas al déficit democrático de la UE y a su gobierno tecnocrático ${ }^{12}$. Sin embargo, lo que está en juego no es ya sólo cómo democratizar a la Unión, sino si ello es posible a nivel supranacional; es decir, la posibilidad de exportar el lenguaje moderno de la democracia liberal -representación, soberanía, demos, legitimación, rendimiento de cuentas- a una nueva ontología política que trasciende las fronteras de los estados nacionales sin eliminarlas.

\footnotetext{
${ }_{9}$ Véase la versión consolidad del Tratado de la UE, Diario Oficial de la Unión Europea, C 83/13, de 30/3/2010, https://www.boe.es/doue/2010/083/Z00013-00046.pdf (02/10/2018). Sobre la forma de gobierno de la UE véase Marta Postigo, "Beyond the Nation-State: The European Union and Supranational Democracy", Varieties of Liberalism. Contemporary Challenges, en Jan Harald Alnes y Manuel Toscano, eds. Newcastle upon Tyne: Cambridge Scholars Publishing, 2014, 172-190.

${ }^{10}$ Para una reflexión más amplia sobre la idea de una nación o un pueblo europeo véase, por ejemplo, Anders Hellström, Bringing Europe Down to Earth. Lunds Universtet, 2006. LACEY, Joseph (2016): "Conceptually Mapping the European Union: A Demoi-cratic Analysis", Journal of European Integration, Vol. 38, Issue 1, 61-77.

${ }^{11}$ La literatura académica sobre demoicracia europea es amplia y ha crecido en los últimos ańos, siendo éste uno de los conceptos y de las categorías discutidos en los estudios contemporáneos de la UE. Algunos de los autores más representativos de dicho debate son Richard Bellamy y Dario Castiglione, "Three Models of Democracy, Political Community and Representation in the EU", Journal of European Public Policy 20:2 (2013), 206-223; Richard Bellay, "An Ever Closer Union among the Peoples of Europe: Republican Intergovernmentalism and Demoicratic Representation within the EU, Journal of European Integration, Vol. 35, Num. 5 (2013), 419-516; F. Cheneval y F. Schmmelfennig, "The Case for Demoi-cracy in the European Union, Journal of Common Market Studies, 51, Num. 2 (2013), 334-350; F. Cheneval, S. Lavenex y F. Schmmelfennig, "Demoi-cracy in the European Union: Principles, Institutions, Policies, Journal of European Public Policy, Vol. 22, Num. 18 (2015), 1-18; K. Nicolaïdis, "The Idea of a European Demoi-cracy", Philosophical Foundations of European Union. J. Dickinson y P. Eleftheriadis, eds. Oxford: Oxford University Press, 2012, 247-274; K. Nicolaïdis, "Pragmatism, Idealism and European Demoicracy", Social Europe Journal, 15 de julio de 2013: https://www.socialeurope.eu/2013/07/pragmatism-idealism-and-european-democracy/ (15/03/2017).

12 Jürgen Habermas, The Lure of Technocracy. Cambridge: Polity Press, 2015; Vivien Schmidt, "Democratizing the Eurozone", Social Europe Journal, 5 de mayo de 2013, en línea: http://www.social-europe.eu/2012/05/ democratizing-the-eurozone/?utm_source=feedburner\&utm_medium=feed\&utm_campaign=Feed\%3A+social-europe\%2FwmyH+\%28Social+Europe+Journal\%29 (21/05/2013).
} 
¿Es posible trasladar las categorías, los principios y las instituciones de la democracia representativa moderna a la nueva ontología política comunitaria? ¿O ésta última conlleva una reinvención y adaptación del léxico cívico y político en un nuevo marco político?

Cabría responder que las democracias representativas han sido concebidas en el marco de los estados nacionales, y que las instituciones de gobierno supranacionales, salvo la Comisión Europea, rinden cuentas a los electorados nacionales, como indica el concepto de demoicracia. Sin embargo, lo cierto es que dicha interpretación ignora el rasgo distintivo de la UE, es decir, su doble dimensión supranacional e interestatal.

La primera, la supranacional, está encarnada en el Parlamento Europeo, elegido por sufragio universal directo de la ciudadanía desde 1979. Como se verá más adelante, la ciudadanía de la Unión, definida formalmente por primera vez en el Tratado de Maastricht, refleja la aspiración cívica del proyecto de integración y la creación de un espacio cívico-político supranacional que trasciende, si eliminarlas, a las fronteras nacionales.

Sin embargo, junto la ciudadanía de la Unión, el Consejo Europeo y el Consejo ponen de relieve la dimensión interestatal e intergubernamental que caracteriza al proceso de toma de decisiones comunitario. En ellos, se tienen en cuenta los intereses de los Estados Miembros, representados por sus Jefes de Estado o de Gobierno y sus Ministros, que rinden cuentas ante los electores y los Parlamentos nacionales.

Por ello, no es correcto adoptar la perspectiva del estado-nacional para definir a la Unión, pero tampoco basta con equipararla con una organización supranacional o internacional como el Consejo de Europa y las Naciones Unidas que carecen de un Parlamento propio democráticamente electo por la ciudadanía y de un estatus cívico independiente y superpuesto al nacional. Resulta pertinente preguntarse, al fin y al cabo, en qué medida es posible democratizar una entidad política no-estatal como la UE ${ }^{13}$.

Aun así, y aunque pueda parecer exagerado apelar a un demos Europeo, lo cierto es que existe una ciudadanía comunitaria que pone de relieve el alcance cívico y político del proyecto de integración. Por este motivo, y por su relevancia para pensar el futuro del proyecto comunitario, resulta pertinente detenerse en las características de la ciudadanía europea, así como en la estructura jurídica multinivel que tiene lugar en la Unión.

\footnotetext{
${ }_{13}$ Schmitter, How to Democratize the European Union - and Why Bother?, op. cit.; Schmitter y Treschel, "The Future of Democracy in Europe: Trends, Analysis and Reforms", op. cit.
} 


\section{Los desafíos de la ciudadanía europea: libre circulación, inmigración y cosmopolitismo.}

UNO DE LOS LOGROS DE LA UE que refleja de la dimensión cívica del proyecto comunitario es la creación de la ciudadanía europea. Ésta se enfrenta, no obstante, a desafíos políticos y conceptuales que merece la pena analizar para pensar el futuro de la Unión y también el camino por el que puede avanzar una ciudadanía cosmopolita en el siglo XXI.

El artículo 20 del Tratado sobre el Funcionamiento de la Unión Europea (TFUE) y el artículo 9 del TUE establecen la ciudadanía supranacional en los siguientes términos: "será ciudadano de la Unión toda persona que ostente la nacionalidad de un Estado miembro. La ciudadanía de la Unión se añade a la ciudadanía nacional sin sustituirla" ${ }^{14}$.

A pesar de la brevedad de dicha formulación, de apenas dos líneas, la misma da lugar a reflexiones y controversias que merece la pena explorar. En primer lugar, la primera parte de la definición pone de relieve el carácter derivativo de la ciudadanía europea, esto es, su dependencia o derivación de la nacionalidad de los Estados Miembros. Desde esta perspectiva podría parecer que la ciudadanía de la Unión no trasciende significativamente la dimensión nacional ni plantea un reto a la hegemonía de los estados. Éstos, los Estados Miembros, mantienen sus competencias y prerrogativas en materia de nacionalidad, de ciudadanía, de naturalización e inmigración, y en general, en aquellas áreas y políticas en las que resultan más eficientes -el principio de subsidiariedad-. En tanto que el requisito para acceder a la ciudadanía europea es ostentar la nacionalidad de uno de los Estados Miembros, cabría afirmar que la ciudadanía comunitaria no añade nada nuevo al paradigma o al dominio nacional.

Sin embargo, lo cierto es que la doctrina del Tribunal de Justicia de la Unión Europea (TJUE) y la literatura académica han puesto de relieve en los últimos años que el carácter derivativo de la ciudadanía europea no anula su dimensión autónoma y supranacional ${ }^{15}$. Al contrario, cabe afirmar que "la ciudadanía de la UE crea

14 Véase la versión consolidad del TFUE, Diario Oficial de la Unión Europea, 30/03/2010, C 87/47, https://www. boe.es/doue/2010/083/Z00047-00199.pdf (02/10/2018).

15 J. Shaw, "The Interpretation of European Union Citizenship", The Modern Law Review, 61, 3 (1998), 293-317; Dimitry Kochenov, "The Essence of EU Citizenship Emerging from the Last Ten Years of Academic Debate: Beyond the Cherry Blossoms and the Moon?", International and Comparative Law Quarterly, Vol 62, Issue 1 (January, 2013), 97-136; Dimitry Kochenov, “The Citizenship of Personal Circumstances in Europe”, Questioning EU Citizenship. Judges and the Limits of Free Movement and Solidarity in the EU, Daniel Thym, ed. Oford y Portland, Orgeon: Bloomsbury, 2017, 51-81; Dora Kostakopoulou, "Ideas, Norms and European Citizenship: Explaining Institutional Change”, Modern Law Review, 68 (2) (2005), 233-267; Dora Kostakopoulou, "European Union Citizenship: Writing the Future", European Law Journal, Vol. 13, Num. 5 (2007), 623-646; 
una esfera política nueva que está 'por encima' de la de los Estados Miembros y cuyos sujetos, los ciudadanos europeos, tienen derechos y un estatus que trasciende al de los Estados Miembros" ${ }^{16}$.

Asimismo, teniendo en cuenta la segunda parte de la definición, esto es, que "la ciudadanía de la Unión se añade a la ciudadanía nacional sin reemplazarla", es posible advertir que en el ámbito comunitario coexisten dos estatus cívicos o doble ciudadanía, la nacional y la supranacional. El doble estatus cívico o la ciudadanía dual, nacional y supranacional, a la que ha dado lugar la Unión, no tiene precedentes ni equivalentes en otras regiones u organizaciones supranacionales.

Reflexionar sobre sus implicaciones conceptuales, cívicas y políticas, es un objetivo relevante, por tanto, para entender los caminos por los que podría avanzar una ciudadanía cosmopolita en el siglo xxI.

Aunque algunos autores y autoras consideran que el acceso a la ciudadanía europea debería desligarse por completo de la nacionalidad y pasar a depender de otro criterio como la residencia ${ }^{17}$, ello parece no solo poco probable a la luz de las derivas antieuropeas y antiinmigración que están proliferando en el continente, sino que supondría una pérdida de su carácter dual; de lo que Beck y Grande denominan la lógica bothland (tanto nacional como supranacional) ${ }^{18}$.

En general, puede decirse que la Unión se ha convertido "en el hogar de la forma más avanzada de ciudadanía multinivel que tiene lugar en el mundo hoy en día, anticipando otros desarrollos parecidos en otros lugares de integración regional" 19 .

Entender el alcance y el significado de la ciudadanía europea, así como su carácter autónomo, implica conocer, asimismo, los derechos que ésta otorga, pero también los deberes que plantea a los Estados Miembros, y por tanto, los límites que supone para la soberanía nacional. Ciertamente, los estados han de adaptar sus ordenamientos jurídicos para cumplir con el derecho europeo de ciudadanía.

Tales consideraciones conducen a la siguiente pregunta: ¿qué objeciones cabe plantear a un estatus añadido de ciudadanía que se concede de forma automática a los nacionales de los Estados Miembros, sin sustituir ni anular a su nacionalidad ni

\footnotetext{
Willem Maaas, "The Origins, Evolution and Political Objectives of EU Citizenship”, German Law Journal, Vol. 15, Num. 5 (2014), 797-820; Willem Maas, "European Governance of Citizenship and Nationality", Journal of Contemporary European Research, Vol. 12, Num. 1 (2016), 533-551; Stefanie Pukallus, Representations of European Citizenship since 1951. London: Palgrave Macmillan, 2016.

16 Maas, "European Governance of Citizenship and Nationality", op. cit., 544.

${ }_{17}$ Kostakopoulou, "European Union Citizenship: Writing the Future”, op. cit., 644-645; Maas, "European Governance of Citizenship and Nationality”, op. cit., 538.

18 Beck y Grande, Cosmopolitan Europe, op. cit.

19 Maas, "European Governance of Citizenship and Nationality", op. cit., 544.
} 
a sus derechos, sin exigirles en principio méritos o virtudes específicas, y que amplía sus oportunidades y garantías jurídicas?

Lo cierto es que la retórica política es hábil para convertir los beneficios en riesgos y presentar a la ciudadanía europea como una pérdida en lugar de como una ganancia, como puede verse en el caso del Brexit.

Son cuatro los derechos básicos que confiere automáticamente la ciudadanía comunitaria, tal y como son definidos en el artículo 20.1. TFUE: 1) el derecho de circular y residir libremente en el territorio de los Estados Miembros; 2) el sufragio activo y pasivo en las elecciones al Parlamento Europeo y en las elecciones municipales del Estado Miembroen el que se reside, en las mismas condiciones que los nacionales de dicho estado; 3) el derecho de acogerse, en el territorio de un tercer país en el que no esté presentado el Estado Miembrode procedencia, a la protección de las autoridades diplomáticas y consultares de cualquier Estado Miembroen las mismas condiciones que los nacionales de dicho estado; 4) el derecho de formular peticiones al Parlamento Europeo, de recurrir al Defensor del Pueblo Europeo, así como de dirigirse a las instituciones y a los órganos consultivos de la Unión en una de las lenguas de los Tratados y de recibir una contestación en esa misma lengua.

Se trata, como puede apreciarse, de cuatro derechos que amplían el espacio político y cívico comunitario. De éstos, la libertad de circulación y de residencia en el territorio de la Unión es el que la ciudadanía identifica de forma más directa con el estatus cívico supranacional y con los beneficios que trae consigo la integración ${ }^{20}$.

Sin embargo, dicho logro, que constituye un derecho fundamental reforzado por la doctrina del TJUE y el cuarto pilar de la Unión junto a la libre circulación de bienes, de capitales y de servicios, es el que encuentra más obstáculos legales y políticos en su implementación ${ }^{21}$.

Como se ha indicado, la regulación del derecho de libre circulación y de residencia pone en evidencia la paradoja "entre el derecho fundamental y sus excepciones" 22 . Junto a ello, las ambigüedades conceptuales y normativas del derecho de movilidad, que acentúan el protagonismo del TJUE en asuntos políticos, y los obstáculos que plantean los discursos antieuropeos y antiinmigración a la ciudadanía comunitaria, merecen un amplio análisis que, en las páginas que siguen, sólo puede tratarse de forma sucinta, destacando sus rasgos fundamentales.

\footnotetext{
${ }^{20}$ Véanse Comisión Europea, EU Citizenship Report 2013. Bruselas, 2013, http://ec.europa.eu/justice/citizen/ files/2013eucitizenshipreport_en.pdf (17/10/2014); Shaw, "The Interpretation of European Union Citizenship", op. cit., 303; Maas "The Origins, Evolution and Political Objectives of EU Citizenship", op. cit., 818-819.

${ }^{21}$ Daniel Thym, "Introduction: The Judicial Deconstruction of Union Citizenship", Daniel Thym, ed., Questioning EU Citizenship. Judges and the Limits of Free Movement and Solidarity in the EU, op. cit., 10-27.

${ }_{22}$ Ídem.
} 
En primer lugar, cabe reflexionar sobre las excepciones formalmente completadas a la libertad de circulación y residencia en la UE. De acuerdo con la Directiva 2004/38/CE del Parlamento Europeo y del Consejo, de 29 de abril de 2004, relativa al derecho de los ciudadanos de la Unión y de los miembros de sus familias a circular y residir libremente en el territorio de los Estados Miembros ${ }^{23}$, estos últimos, los estados, pueden poner restricciones temporales a la libre circulación de personas por razones de "seguridad pública", "salud pública", "política pública" y "empleabilidad en sector público". Junto a ésta, la Convención de Schengen permite a los estados parte reestablecer sus controles fronterizos, internos y externos, e imponer restricciones a la movilidad por períodos limitados en casos de "seguridad nacional" 24 .

Sin embargo, no son tales restricciones temporales y excepcionales a la libre circulación de personas dentro de la Unión las que plantean mayores controversias. Por el contrario, lo son las limitaciones que encuentran los ciudadanos y las ciudadanas desempleados o sin recursos suficientes para "no convertirse en una carga excesiva", quienes, en la práctica, no tienen garantizados plenamente su derecho fundamental a la libertad de movilidad y de residencia, y desafían el carácter universalista de la ciudadanía comunitaria.

Dicho de otra forma, son los ciudadanos con empleo o con recursos suficientes para mantenerse por sí mismos y no resultar una "carga excesiva" para el estado receptor quienes gozan de plenos derechos de movilidad y de residencia en la UE. Por este motivo, resulta cuestionable que la ciudadanía europea se haya convertido en un estatus independiente de los objetivos económicos y del mercado interno.

Para entender mejor dichas objeciones, conviene conocer las distinciones que establece la Directiva 2004/38/CE que regula el derecho fundamental de circulación y residencia dentro de la Unión en función del tiempo de residencia en un Estado Miembro diferente al de origen. Así, durante los tres primeros meses, los ciudadanos europeos pueden residir libremente en otro Estado Miembro sin que les sean exigidos requisitos. Tras cinco años de residencia legal y continuada en un Estado Miembro extranjero, los ciudadanos y las ciudadanas de la Unión pueden

${ }^{23}$ Directiva 2004/38/EC of the European Parliament and the Council, of 29 April 2004, Official Journal of the European Union, L 158, 30-4-2004.

${ }^{24}$ El "espacio Schengen" fue creado en 1985 en virtud del acuerdo alcanzado entre cinco países europeos, Bélgica, Alemania, Francia, Luxemburgo y los Países Bajos, que firmaron el Convenio de Aplicación en junio de 1990 (en vigor en 1995). Dicho espacio, que regula la movilidad libre de visados y refuerza la cooperación en seguridad intra- $\mathrm{y}$ transfronteriza, está integrado actualmente por los veintiséis países europeos, de los que veintidós son miembros de la UE: Bélgica, Chequia, Dinamarca, Alemania, Estonia, Grecia, Espańa, Francia, Italia, Letonia, Lituania, Luxemburgo, Hungría, Malta, Los Países Bajos, Austria, Polonia, Portugal, Eslovenia, Eslovaquia, Finlandia y Suecia, así como Islandia, Liechtenstein, Noruega y Suiza. Por su parte, hasta la fecha, Bulgaria, Croacia, Chipre, Irlanda, Rumanía y el Reino Unido no forman parte del espacio Schengen. 
acceder a la residencia permanente, lo que implica los mismos derechos en virtud del principio de no discriminación e igualdad de trato.

Sin embargo, en el período intermedio, esto es, pasados los tres primeros meses y antes de los cinco ańos, para permanecer en un Estado Miembro sin nacionalidad es preciso demostrar que se tienen recursos suficientes y cobertura sanitaria. En otras palabras, los ciudadanos de la Unión pueden residir libremente en otro Estado Miembro antes de los cinco años de residencia legal siempre que no constituyan una carga excesiva para el estado receptor, circunstancia que, no obstante, no dará lugar a una expulsión automática (TJUE, Caso Grzelczyk C184/99)25.

Por tanto, si bien la libre movilidad resulta efectiva durante los tres primeros meses sin distinciones para todos los ciudadanos, y transcurridos los cinco ańos de residencia legal y continuada los derechos se equiparan en todos los aspectos, la normativa europea es ambigua con respecto a los ciudadanos sin recursos y sin empleo cuya capacidad de permanecer en un Estado Miembro extranjero está sujeta a la condición de "no ser una carga" ${ }^{26}$.

Pero, ¿quiénes y cuándo representan los ciudadanos una carga excesiva para un Estado Miembro? ¿'Tienen derecho los ciudadanos sin empleo o sin recursos suficientes a solicitar ayudas sociales no contributivas en el estado receptor antes de adquirir la residencia legal?

En efecto, la controversia básica que plantea actualmente la ciudadanía europea tiene que ver con el acceso a los beneficios y a las ayudas sociales no contributivas en el estado receptor. Otro aspecto que requiere un prologando debate es, sin duda, la acogida de inmigrantes de terceros países que, lamentablemente, no puede abordarse en estas páginas. A este respecto, conviene mencionar tan sólo que, si bien los estados preservan sus prerrogativas en materia de naturalización, de inmigración, de nacionalidad y de ciudadanía, deben adaptar sus ordenamientos jurídicos a la normativa comunitaria y cumplir con los compromisos adoptados a nivel supranacional. De momento será suficiente, no obstante, con destacar los aspectos más controvertidos que afectan a los ciudadanos y a las ciudadanas que ya son europeos y a la inmigración intracomunitaria.

Por un lado, conforme al derecho de la UE y a la jurisprudencia del TJUE, los ciudadanos y las ciudadanas de la Unión tienen garantizado el derecho fundamental de igualdad de trato y de no discriminación por razón de la nacionalidad.

Sin embargo, en los últimos años, los discursos sobre el "turismo social" dentro de la Unión -particularmente desde que tuvieron lugar las ampliaciones a Europa

${ }^{25} \mathrm{La}$ sentencia puede leerse en línea: https://eur-lex.europa.eu/legal-content/EN/TXT/?uri=CELEX\%3A61999CJ0184 (02/10/2018).

${ }^{26}$ Véase Kochenov, “The Citizenship of Personal Circumstances in Europe”, op. cit., 51-80. 
del Este de 2004 y 2007, y también a raíz de la crisis económica-, han estado en el centro de los discursos nacionalistas y populista que caracterizan a los partidos antiinmigración y antieuropeos. El Brexit es un claro reflejo de cómo la ciudadanía europea puede verse como un perjuicio, en lugar de como una ganancia, para los estados que son receptores de inmigrantes intracomunitarios, a los que se reprocha aprovecharse de los derechos que otorga la ciudadanía comunitaria para hacer "turismo social" y abusar de sus generosas prestaciones sociales ${ }^{27}$.

Por su parte, la Directiva 2004/38/CE no excluye explícitamente el acceso a beneficios y prestaciones sociales no contributivas a ciudadanos europeos no nacionales durante los tres primeros meses de residencia. Sin embargo, los Estados Miembros no están obligados a ofrecer asistencia ni beneficios sociales a inmigrantes intracomunitarios que carecen de residencia legal. Como se ha indicado, la Directiva comunitaria menciona hasta ocho veces que los ciudadanos de la Unión no deben convertirse "en una carga para los sistemas de asistencia social del Estado Miembro de acogida", y, de acuerdo con el artículo 7.1. que los estados pueden tomar todas las medidas que resulten necesarias para impedirlo ${ }^{28}$.

Asimismo, tal y como ha puesto de relieve Ferdinand Wollesnchläger ${ }^{29}$, el régimen legal europeo sobre libertad de movimiento contiene ambigüedades que dan margen a amplios debates políticos y jurídicos. Por un lado, para prevenir que los ciudadanos europeos que se trasladan a otro Estado Miembro se conviertan en una carga excesiva para éste se les exige la autosuficiencia económica como criterio para la residencia. Sin embargo, no tener suficientes recursos no implica perder el derecho de residencia y el acceso a ayudas sociales, ni tampoco la expulsión automática. En definitiva, son los estados y los tribunales competentes quienes han de interpretar cuándo un inmigrante intracomunitario constituye una "carga excesiva" para dicho estado receptor, y cuál es el margen de discreción que permite justificar un trato "indirectamente discriminatorio" en base a las cuentas públicas ${ }^{30}$.

\footnotetext{
${ }^{27}$ Susan K., Schmidt, "Extending Citizenship Rights and Losing it All: Brexit and the Perils of 'Over-Constitutionalisation", en Daniel Thym, ed. Questioning EU Citizenship. Judges and the Limits of Free Movement and Solidarity in the EU, op. cit., 28-51; Ferdinand Wollenschläger, "Consolidating Union Citizenship: Residence and Solidarity Rights for Jobseekers and the Economically Inactive in the Post-Dano Era", en Daniel Thym, ed. Questioning EU Citizenship. Judges and the Limits of Free Movement and Solidarity in the EU, op. cit., 210-232.

${ }_{28}$ Schmidt, "Extending Citizenship Rights and Losing it All: Brexit and the Perils of 'Over-Constitutionalisation"”, op. cit., 32.

29 Wollenschläger, "Consolidating Union Citizenship: Residence and Solidarity Rights for Jobseekers and the Economically Inactive in the Post-Dano Era”, op. cit., 210-232.

${ }^{30}$ Puede verse la controvertida sentencia del TJUE, European Commission versus Federal Republic of Germany, Case C-206/10, 5 de mayo de 2011, en la que el Tribunal da la razón a Alemania al requerir residencia legal para acceder a beneficios sociales no contributivos: ECLI: EU:C:2011:283, https://eur-lex.europa.eu/legal-content/ EN/TXT/?uri=CELEX\%3A62010CJ0206 (02/10/2018).
} 
Ciertamente, la capacidad de los Estados Miembros de ayudar o sostener económicamente, con cargo a sus sistemas públicos de bienestar no contributivos, a ciudadanos y ciudadanas no nacionales sin recursos y desempleados, que además no muestran intención de buscar empleo, pone en juego el alcance de la solidaridad. Ahora bien, lo que se discute es la propia universalidad del estatus cívico y de la ciudadanía de la Unión.

A pesar de los esfuerzos por armonizar los sistemas públicos de Seguridad Social en la $\mathrm{UE}^{31}$, no existe a nivel supranacional una única política social. Las controversias políticas y sociales en torno a la movilidad y a la inmigración intracomunitaria ponen de relieve que la UE se ha construido a través de una legislación minimalista en materia social ${ }^{32}$. No existe a nivel europeo "un concepto uniforme de solidaridad que fundamente el acceso a los beneficios sociales, en tanto que es preciso distinguir entre las ayudas ligadas al empleo y a la asistencia social a la que se accede en los sistemas de bienestar domésticos" 33 .

Por ello, en los últimos años, ha sido el TJUE quien ha jugado un papel decisivo en la definición del alcance y de la extensión del derecho de movilidad y de residencia como derechos fundamentales de la ciudadanía europea. A este respecto, sus resoluciones más recientes reflejan un giro más restrictivo en su doctrina, que da la razón a estados como Alemania y el Reino Unido en su negativa a conceder ayudas sociales no contributivas a inmigrantes de dentro de la Unión sin residencia legal en dichos territorios ${ }^{34}$.

Tales hechos suscitan amplios debates académicos y políticos sobre la llamada "judicialización” del proceso de integración europeo, esto es, sobre el papel clave del TJUE en la integración europea mediante su interpretación del alcance del derecho comunitario.

Asimismo, ponen de relieve el impacto de los discursos antieuropeos y antiinmigración, definidos como populistas a mi juicio de forma acertada, a la hora de

\footnotetext{
${ }^{31}$ Pueden verse los Reglamentos CE 883/2004 del Parlamento Europeo y del Consejo, de 29 de abril, sobre la Coordinación de los Sistemas de Seguridad Social (DOUE de 30 de abril) y CE 987/2009 del Parlamento Europeo y del Consejo de 16 de septiembre de 2009, por el que se adoptan las normas de aplicación del Reglamento CE Num. 883/2004, DOUE de 30/10/2009.

${ }^{32}$ Daniel Thym, "The Evolution of Citizens' Rights in Light of the European Union's Constitutional Development", en Daniel Thym, ed. Questioning EU Citizenship. Judges and the Limits of Free Movement and Solidarity in the EU, op. cit., 148.

33 Ídem.

${ }^{34}$ Me refiero a las sentencias del TJUE European Commission versus Federal Republic of Germany, Case C-206/10, y Commission versus United Kingdom C-308/14; Véanse Thym, "The Evolution of Citizens' Rights in Light of the European Union's Constitutional Development", op. cit.; Wollenschläger, "Consolidating Union Citizenship: Residence and Solidarity Rights for Jobseekers and the Economically Inactive in the Post-Dano Era”, op. cit., 210-232.
} 
dar por hecho que se produce el llamado "turismo social" y de atribuir desventajas sociales y económicas a la movilidad europea, cuando las evidencias indican lo contrario. Tales retóricas ocultan frecuentemente los beneficios, no sólo económicos, sino cívicos que trae consigo la movilidad dentro de la $\mathrm{UE}^{35}$.

En general, las consideraciones previas muestran las controversias que suscita la integración cívica europea. ¿Están justificadas las restricciones a la ciudadanía europea para proteger o para recuperar la soberanía democrática de los Estados Miembros? ¿Cómo conciliar el proyecto cívico cosmopolita y la soberanía nacional en materia de fronteras e inmigración? ¿Debe ofrecer la UE una respuesta coordinada y uniforme al fenómeno general de la inmigración de terceros países y al problema de las fronteras exteriores europeas?

Tales cuestiones, y particularmente el fenómeno migratorio, resultan decisivas para pensar el futuro, no sólo del proyecto europeo, sino de la ciudadanía del siglo XXI. Que triunfe el cosmopolitismo o el nacionalismo es algo que todavía no podemos saber con plena certeza. En todo caso, la UE es un experimento cívico y político que puede resultar clave para comprender cómo pueden formalizarse un doble estatus de ciudadanía y un proyecto cívico supranacional.

\footnotetext{
35 Veanse "Labour Mobility Within the EU: The Real Picture", Social Europe Journal, 12 de noviembre de 2014, https://www.socialeurope.eu/2014/11/labour-mobility-within-eu-real-picture/ (25/08/2016), y Comisión Europea, Informe de la Comisión al Parlamento Europeo, al Consejo, al Comité Económico y Social Europeo y al Comité de las Regiones elaborado en aplicación del Artículo 25 del TFUE sobre progreso hacia el ejercicio efectivo de la ciudadanía de la Unión durante el período 2011-2013, COM/2013/0270 final: http://eur-lex. europa.eu/legal-content/ES/TXT/?uri=celex:52013DC0270 (21/12/2017);
} 


\section{REFERENCIAS BibLIOGRÁficAS}

Anheier, Helmut K., Mary Kaldor y Marlies Gasious, eds., Global Civil Society 2005/6. Londres, SAGE Publications, 2006.

BeCK, UlRICH, Was ist Globalisierung? Irrtümer des Globalismus - Antworten auf Globalisierung. Frankfurt am Main, Suhrkamp, 1997.

- Cosmopolitan Vision. Cambridge: Polity Press, 2006.

Beck, Urlich y Edgar Grande, Cosmopolitan Europe. Cambridge, Polity Press, 2007.

Bellamy, Richard y Dario Castiglione, "Three Models of Democracy, Political Community and Representation in the EU", Journal of European Public Policy, 20:2 (2013), 206-223.

Bellamy, Richard, "An Ever Closer Union among the Peoples of Europe: Republican Intergovernmentalism and Demoicratic Representation within the EU, Journal of European Integration, Vol. 35, Num. 5 (2013), 419-516.

Benhabib, Seyla, The Rights of Others. Aliens, Residents and Citizens. Cambridge, Cambridge University Press, 2004.

Benhabib, Benhabib, Ian Shapiro y Danilo Patronovic, eds., Identities, Affiliations, and Allegiancies. Cambridge, Cambridge University Press, 2007.

Benhabib, Seyla, Another Cosmopolitanism. Robert Post, ed. Oxford, Oxford University Press, 2006.

Burgess, Michael, Federalism and the European Union: The Building of Europe, 1950-2000. Londres, Routledge, 2000.

Castells, Castells, The Rise of the Network Society. The Information Age: Economy, Society and Culture. Vol. I. Cambridge, MA, Oxford, UK, Blackwell, 1997.

Cheneval, Francis y F. Schmmelfennig, "The Case for Demoi-cracy in the European Union, Journal of Common Market Studies, 51, Num. 2 (2013), 334350.

Cheneval, Francis, S. Lavenex y F. Schmmelfennig, "Demoi-cracy in the European Union: Principles, Institutions, Policies, Journal of European Public Policy, Vol. 22, Num. 18 (2015), 1-18.

Castaldi, Roreberto, "A Federalist Framework Theory on European Integration", Centro Studi Federalismo, 2007,1-93. 
Comisión Europea, EU Citizenship Report 2013. Bruselas, 2013, http://ec.europa.eu/justice/citizen/files/2013eucitizenshipreport_en.pdf (17/10/2014).

Costa, Oliver, "The Coming of Age of the European Parliament", Journal of European Integration, 8 de marzo de 2017, http://www.tandfonline.com/doi/ful 1/10.1080/07036337.2017.1297021 (08/03/2017).

Dosenrode, Soren, "Federalism Theory and Neo-functionalism: Elements for an Analytical Framework", Perspectives on Federalism, Vol. 2, Issue 3 (2010), $1-28$.

Elazar, Daniel J., Exploring Federalism. Londres, The University of Alabama Press, 1987.

Fabbrini, Mario, "Transatlantic Constitutionalism: Comparing the United States and the European Union", European Journal of Political Research, 43 (2004), 547-569.

- "The European Multilevel System for the Protection of Fundamental Rights: A 'Neo-Federalist Perspective', Jean Monnet Working Paper, 15/10 (2010).

Friedman, Thomas L., The World is Flat. A Brief History of the Twenty-First Century. New York, Picador-Farrar, Straus y Giroux, 2005.

Habermas, Jürgen, Die postnationale Konstellation: Politische Essays. Frankfurt, Suhrkamp Verlag, 1998.

- The Lure of Technocracy. Cambridge, Polity Press, 2015.

Held, David y Anthony McGrew, Globalization/Anti-Globalization. Cambridge, Polity Press, 2002.

Hellström, Anders, Bringing Europe Down to Earth. Lunds Universtet, 2006.

Kaldor, Mary Kaldor, Global Civil Society An Answer to War. Cambridge: Polity Press, 2003.

Keane, John, ¿Global Civil Society? Cambridge, Cambridge University Press, 2003.

Kochenov, Dimitry, "The Essence of EU Citizenship Emerging from the Last Ten Years of Academic Debate:Beyond the Cherry Blossoms and the Moon?", International and Comparative Law Quarterly, Vol 62, Núm. 1 (2013), 97-136. 
- "The Citizenship of Personal Circumstances in Europe", Questioning EU Citizenship. Judges and the Limits of Free Movement and Solidarity in the EU, en Daniel Thym, ed. Oxford y Portland, Oregon, Bloomsbury, 2017, 51-81.

Kostakopoulou, Dora, "Ideas, Norms and European Citizenship: Explaining Institutional Change”, Modern Law Review, 68 (2) (2005), 233-267.

- "European Union Citizenship: Writing the Future", European Law Journal, Vol. 13, Num. 5 (2007), 623-646.

Lacey, Joseph, "Conceptually Mapping the European Union: A Demoi-cratic Analysis", Journal of European Integration, Vol. 38, Num. 1 (2016), 61-77.

Maas, Willem, "The Origins, Evolution and Political Objectives of EU Citizenship", German Law Journal, Vol. 15, Num. 5 (2014), 797-820.

- "European Governance of Citizenship and Nationality", Journal of Contemporary European Research, Vol. 12, Num. 1 (2016), 533-551.

NicolaÏDis, Kalypso, "Pragmatism, Idealism and European Demoicracy", Social Europe Journal, 15 de julio de 2013, https://www.socialeurope.eu/2013/07/ pragmatism-idealism-and-european-democracy/ (15/03/2017).

- "The Idea of a European Demoi-cracy", Philosophical Foundations of European Union, J. Dickinson y P. Eleftheriadis, eds. Oxford: Oxford University Press, 2012, 247-274.

Praino, Diego, "A New System of Government? Defining the Confidence Relationship of the EU Model", Journal of European Integration, 01 de Febrero de 2017, http://www.tandfonline.com/doi/full/10.1080/07036337.2017.128126 $4(08 / 03 / 2017)$.

Pukallus, Stefanie, Representations of European Citizenship since 1951. Londres, Palgrave Macmillan, 2016.

Shaw, Jo, "The Interpretation of European Union Citizenship", The Modern Law Review, 61, 3 (1998), 293-317.

Schmidt, Vivien, "Democratizing the Eurozone", Social Europe Journal, 5 de mayo de 2013, en línea: http://www.social-europe.eu/2012/05/democratizing-the-eurozone/?utm_source=feedburner\&utm_medium=feed \&utm_campaign=Feed\%3A+social-europe\%2FwmyH+\%28Social+Europe+Journal\%29 (21/05/2013).

Schmidt, Susan K., "Extending Citizenship Rights and Losing it All: Brexit and the Perils of 'Over-Constitutionalisation'", en Questioning EU Citizenship. Judges and the Limits of Free Movement and Solidarity in the EU, Daniel Thym, ed. Oxford y Portland, Oregon, Bloomsbury, 2017, 28-51. 
Schmitter, Philippe, "What Is There to Legitimize in the European Union... and How Might This be Accomplished?", European University Institute (enero 2001), en-línea: http://www.eui.eu/Documents/Departments-Centres/SPS/ Profiles/Schmitter/LegitimizeEU.pdf, (08/11/2012).

Schmitter, Philippe y Alexander H. Treschel, coords., "The Future of Democracy in Europe: Trends, Analysis and Reforms". A Green Paper for the Council of Europe. Council of Europe, 2007, en línea http://www.thefutureofrepresentativedemocracy.org/files/pdf/resources/schmitter_thefutureofDemocracyinEurope.pdf (09/11/2012).

Schuman, Robert, Declaración de Robert Schuman, 9 de mayo de 1950, accesible en línea https://europa.eu/european-union/about-eu/symbols/europe-day/ schuman-declaration_es (05/05/2018).

Thym, Daniel, "Introduction: The Judicial Deconstruction of Union Citizenship", en Questioning EU Citizenship. Judges and the Limits of Free Movement and Solidarity in the EU, Daniel Thym, ed. Oxford y Portland, Oregon, Bloomsbury, 2017,10-27.

Wollenschläger, Ferdinand, "Consolidating Union Citizenship: Residence and Solidarity Rights for Jobseekers and the Economically Inactive in the Post-Dano Era”, en Questioning EU Citizenship. Judges and the Limits of Free Movement and Solidarity in the EU, Daniel Thym, ed. Oxford y Portland, Oregon, Bloomsbury, 2017, 210-232.

DOI: http://doi.org/10.15366/bp2019.20.009

Bajo Palabra. II Época. No20. Pgs: 161-180 
